\title{
INTEGRACIÓN E IDENTIDAD: UN PROYECTO HACIA LA COHESIÓN SOCIAL
}

\author{
ALBERTO ARES MATEOS \\ Universidad Pontificia Comillas
}

\begin{abstract}
RESUMEN: La construcción de la identidad es un elemento constitutivo en el desarrollo vital de las personas y de los colectivos. En nuestro mundo actual globalizado y diverso, este elemento cobra una relevancia nuclear, más si cabe desde una mirada de los flujos migratorios y la integración social. Es en el día a día donde se juegan muchas de las dinámicas identitarias, en las que se entrelazan procesos de identidad personal y colectiva, tendencias más estáticas y dinámicas. El objetivo de este trabajo es aportar luz a los modelos de integración y de cohesión social desde los procesos de construcción identitaria, en diálogo con el modelo de integración mixto (MIM). Para este fin, se ha presentado muy enriquecedor el dialogo entre el modelo de conductas mayoritarias y minoritarias de J.L. Segundo, el enfoque de las capacidades de A. Sen, los modelos clásicos de integración de personas inmigrantes, el modelo de construcción de identidades de M. Castells, junto al estudio empírico realizado por P. Norris.
\end{abstract}

PALABRAS CLAVE: identidad; integración; migraciones; cohesión social; globalización.

\section{Integration and identity: A project towards social cohesion}

ABSTRACT: The construction of identity is a vital constituent element in the development of individuals and groups. In our current globalized and diverse world, this element takes on a nuclear relevance, more if possible, from a viewpoint of migratory flows and social integration. It is in daily life that many of the identity dynamics are played, in which processes of personal and collective identity, more static and dynamic tendencies are intertwined. The objective of this work is to provide light to integration and social cohesion models from the processes of identity construction, in dialogue with the mixed integration model (MIM). To this end, the dialogue between Segundo's model of majority and minority behaviors, Sen's capability approach, the classic models of integration of immigrants, Castell's model of construction of identities, as well as the empirical study by P. Norris, has been very enriching.

KEY WORDS: identity; integration; migration; social cohesion; globalization.

\section{INTRODUCCIÓN}

«La transformación de nuestra cultura y nuestra sociedad tendrá que ocurrir a diversos niveles. Si sólo sucediera en las mentes de los individuos (como ya ha pasado en cierta medida), sería impotente. Si obedeciera sólo a la iniciativa del estado, sería tiránica. La transformación personal en grandes números es esencial, y no debe ser sólo una transformación de la conciencia, sino que también ha de implicar la acción individual. Pero los individuos necesitan el alimento de los grupos que llevan consigo una tradición moral que refuerza sus propias aspiraciones». (Bellah, 1985)

La construcción de la identidad es un elemento básico en el desarrollo de la vida de una persona o un colectivo. Entender sus mecanismos y clarificar sus procesos son puntos básicos para comprender cómo las personas conformamos nuestras identidades, y en nuestro caso, especialmente entre las personas que emigran y que comparten un contexto cultural nuevo (Labrador, 2001). 
El objetivo de este trabajo es aportar luz a los modelos de integración y de cohesión social desde los procesos de creación de la propia identidad y la propuesta del modelo de integración mixto (MIM) (Ares, 2017). Para este fin, se ha presentado muy enriquecedor el dialogo entre el modelo de conductas mayoritarias y minoritarias (Segundo, 1973), el enfoque de las capacidades (Sen, 2000), los modelos clásicos de integración de personas inmigrantes, el modelo de construcción de identidades de Castells (2000), junto al estudio empírico realizado por Norris (2003).

En primer lugar, se introducirá el concepto de identidad atendiendo a varias clasificaciones dentro de la gestión de la diversidad. Seguidamente se expondrá la situación de crisis que viven las identidades nacionales en nuestro mundo globalizado y se presentarán dos de las alternativas que se han planteado en el ámbito local (identidad étnica) y global (identidad cosmopolita). Asimismo, se analizarán algunos datos significativos para la construcción de identidades de la mano de un estudio realizado por la profesora Pippa Norris (2003) y se expondrá brevemente el modelo de identidades colectivas de Manuel Castells (2000). Seguidamente, se presentará el modelo mixto de integración desde el modelo de conductas mayoritarias y minoritarias de Juan Luis Segundo (1973). Finalmente, se aportarán algunas conclusiones o guías para seguir el camino.

\section{HABLANDO DE IDENTIDAD}

La primera cuestión que cabe plantearse es el origen ontológico de la identidad. ¿Es la identidad un elemento esencial al ser humano o se construye culturalmente? ¿Se hereda de padres a hijos o se va cimentando en el encuentro con los otros, dentro de un contexto cultural? ¿Se define estática y unidimensionalmente o de forma abierta y multidimensional?

\subsection{Entre lo personal y lo comunitario}

Una incipiente aproximación sería la distinción entre identidad personal y social. La primera podría entenderse como la concepción o percepción que una persona tiene de sí misma, y que de alguna manera le hace ser quien es. Por su parte, la identidad social podría entenderse como el sentido de identificación y de pertenencia a un grupo, como diferenciado de otros.

\subsection{Identidad estática vs identidad dinámica}

La comprensión esencialista de la identidad plantea una comprensión homogeneizadora del modelo cultural y social. Se heredan unos patrones identitarios fuertemente homogeneizadores que se comparten con otros a los cuales les han transmitido esa misma identidad (ya sea de la misma etnia, grupo o nación). Esta identidad se presenta como invariable, unidimensional y dotada de homogeneidad. 
La comprensión dinámica cree que el ser humano construye su identidad en el contacto con los otros y en su interacción con nuestro entorno social y cultural. Desde esta perspectiva, la persona se entiende como una unidad compleja y abierta. El mundo y la cultura se definen básicamente desde lo dinámico y multidimensional.

\subsection{La gestión de la diversidad}

Desde la postura esencialista, lo diverso genera miedo ya que significa acoger un patrón diferente al propio, lo que supone fragmentar o poner en cuestión la propia identidad. Cuando apoyamos nuestra seguridad en una sola dimensión, lo diverso nos presenta la dificultad de dialogar porque sentimos el riesgo de perder en el juego lo que dota de sentido a nuestras vidas. Reducir la realidad a una sola dimensión predominante lo que genera son mecanismos de exclusión, que a la larga desembocan en consecuencias funestas para la convivencia.

Una mirada a la realidad mundial recuerda que vivimos dentro de la diversidad. Siempre ha existido lo diverso dentro de nuestras culturas. Una diversidad que se encarna en distintos acentos, sabores, costumbres, colores, aromas, etc. Cada uno de nosotros somos seres únicos y por tanto diversos entre sí. Cada persona va construyendo su identidad en relación con el otro (Buber, 1969), se va recreando en lo complejo, en lo múltiple tanto en el ámbito de lo cotidiano, como en el contexto social y se podría decir que en lo global. Por tanto, la identidad necesita de la diversidad, no la excluye. Por esta razón, la diversidad se convierte en condición de posibilidad para la construcción de una identidad que es múltiple, compleja, flexible y abierta (Fitzgerald, 1993: 13).

Es lícito tener miedo ante el otro, ante lo nuevo, lo diverso. Pero, ¿simplificar nuestra vida, nuestra cultura, hacia un horizonte homogéneo y unidimensional es la solución? Sería algo deseable si al final nuestra vida también se simplificara y nos ayudara a vivir en sociedad, pero no es así. La diversidad es algo constitutivo de nuestra existencia, lo queramos o no. Por tanto, es necesario tener el coraje de reconocer las diversas dimensiones de nuestra vida, cayendo en la cuenta de la complejidad de las culturas, de nuestra sociedad y de nuestra existencia (Sen, 2011: 24).

Construir la identidad desde la diversidad genera personas únicas, pero a la vez conocedoras de aquellos elementos que se comparten con otros seres humanos. Una persona que vive desde este punto de vista entiende el camino de construcción de su identidad como una dinámica en proceso, siempre abierta, siempre en recreación.

\section{LA CRISIS DE LAS IDENTIDADES NACIONALES}

Anthony Smith en su libro La identidad nacional (1997) fue de los primeros en hablar de las crisis de las identidades, cuando trataba la relación entre es- 
tado y nación. Para este autor el concepto de estado tiene que ver con un elemento institucional, mientras que el de nación haría referencia a un concepto cultural. La nación se definiría como:

«(...) una población humana que comparte un territorio histórico, memorias comunes y mitos, una cultura estandarizada pública, una economía y movilidad territorial común y derechos y obligaciones legales comunes para todos los miembros de la colectividad». (Smith, 1992b: 60)

Según este planteamiento crítico, como los estados contemporáneos son plurales, es decir, comprenden varias comunidades étnicas en su territorio, no se puede hablar de «estados-nación». Se produce una crisis de la manera como se venía entendiendo la creación de identidades.

Las dos respuestas que a mi modo de ver se han planteado ante la llamada crisis de identidades son principalmente dos: la identidad local y la identidad global. Por lo tanto, existe una convivencia de estos tres tipos de identidades: la nacional, la local y la global.

\subsection{La identidad nacional}

La crisis de los estados-nación es un hecho que no podemos obviar. Los ideales de la modernidad, en los que el sentimiento de pertenencia a una unidad nacional se convirtió en un pilar de la identidad social, hoy en día han dejado de tener el mismo sentido (Martínez, 2007: 96-104).

«Una cosa particularmente clara es que el rol y las funciones de los Estados nacionales han cambiado en este nuevo contexto. No es cierto que haya desaparecido, o que en el horizonte esté cercana la pérdida de todo su poder, pero sí que han perdido el control sobre los sectores y decisiones clave de la economía: sobre las corporaciones transnacionales, los precios sobre las materias primas, los flujos de capital o de la información económica, incluso sobre sus propias monedas». (Martínez, 2007: 61)

Pero, ¿por qué antes sí tuvo sentido hablar de estados-nación y ahora no? Gellner (1988) y Smith (1997), entre otros, han explicado este fenómeno. Podemos decir que la creación de estos estados tuvo que ver en gran medida con la creación e institucionalización a través de la educación de una historia, política y cultura común de carácter nacional. Asimismo, se llegó a adoptar una identidad común por la imposición de una étnica prevalente entre las demás, o por la creación de una nueva que aglutinaba a todas. Baumann (2001: 45) expone cómo los estados-nación intentaron convertirse en una "superetnia», situación que no en todos los casos tuvo igual suerte.

Lo que parece un hecho en nuestros días, como apunta Castells (2000: 54), es que: «reducir las naciones y nacionalismos al proceso de construcción del estado-nación hace imposible explicar el ascenso simultáneo del nacionalismo y el declive del estado moderno».

El hecho de la crisis de las identidades nacionales, no significa que estas ya no tengan ningún sentido. Lo que ocurre es que la identidad nacional a su vez 
tiene que convivir con otras fuentes de sentido que en ocasiones trasciende a esta (identidad global), y en otras se sumerge en su pluralidad (identidad local). Este fenómeno es el que Roland Robertson bautizó como «glocalización» (2003).

Robertson populariza este término en los noventa del siglo pasado, tomado del ámbito económico en los ochenta en las empresas japonesas: «Piensa globalmente y actúa localmente». Otros autores como Beck (1998: 79) lo han extendido a otros espacios.

Lo que Robertson plantea es que la globalización no puede ser entendida sólo en términos globales, sino que también tiene que integrar lo local. La globalización presenta el binomio «homogeneización global» $\mathrm{y}$ «heterogeneidad», un debate ya clásico en otras esferas pero que ahora toma tintes planetarios. Diversas áreas de la vida han combinado estos dos ámbitos. Por poner un ejemplo que ilustra este binomio en los modelos de integración, nos encontramos con el asimilacionismo, como polo de homogeneización y universalismo, frente al multiculturalismo, en lo que representa de heterogeneidad y particularismo.

La globalización nos muestra también una nueva caracterización del espacio y el tiempo. La distancia entre ambos se ha ensanchado. Roberson (2003) plantea la irrupción de un tiempo universal, y una abstracción de la presencia física. Estos elementos de la globalización hacen que lo local se construya sobre unas raíces que lo superan (superlocal) y que van más allá de él (translocal).

\subsection{Las identidades locales o étnicas}

La propuesta de las comunidades locales cobra mucha fuerza en nuestras sociedades contemporáneas. Autores como Baumann ven en la etnicidad una eficacia que sobrepasa a la identidad estado-nación.

«La idea de etnicidad tiene una gran ventaja sobre el "estado": nadie necesita un pensamiento abstracto para saber lo que es. La etnicidad implica una serie de raíces: de dónde provengo, qué es lo que me hace ser lo que soy, en una palabra, la identidad natural». (Baumann, 2001: 33).

El elemento de etnicidad presenta un fuerte componente de cohesión social, a la vez que incrementa con creces la capacidad para canalizar una mayor participación colectiva de los individuos.

Es de sobra conocida la convicción de que con la globalización estamos perdiendo control sobre nuestras vidas. No sólo en lo que atañe a los estadosnación, sino a muchos ámbitos y decisiones de nuestra existencia. Los procesos de globalización están plagados de contradicciones de sobra conocidas (Noam, 2004). Asistimos a un mundo cada día más complejo e interconectado donde la supremacía de la economía ha elevado la tendencia hacia la homogeneización cultural (venta de productos en el mercado global), a la exclusión social (de las personas y pueblos que se quedan al margen de forma voluntaria, aunque en la mayoría de los casos involuntariamente) y al individualismo e impersonalización en las relaciones sociales. 
En palabras de Castells, «cuando el mundo se vuelve demasiado grande para ser controlado, los actores sociales pretenden reducirlo de nuevo a su tamaño y alcance» (2000: 89).

«... (Son) las comunas culturales de base religiosa, nacional o territorial (las que) parecen proporcionar la principal alternativa para la construcción de sentido en la sociedad (...) Y aparece entonces la posibilidad del estado local como instancia significativa de reconstrucción del control político y el significado social». (Castells, 2000: 88)

La «glocalización» y el desarrollo de los medios de comunicación de masas, sobre todo Internet, ha propiciado la creación de redes de comunidades étnicas a lo largo y ancho del mundo. Este movimiento de lo étnico o local, frente a lo global, se ha dejado ver en muchos ámbitos de la vida: la política (asociaciones de barrio, partidos políticos locales...), la ecología (producción comunitaria de productos orgánicos...) y los medios de comunicación (periódicos, radios, canales de televisión locales...), entre otros.

\subsection{La identidad global}

\subsubsection{La sociedad de riesgo y la reflexividad del sujeto}

Muchos teóricos de la identidad y/o el cosmopolitismo han visto en la «sociedad de riesgo» un factor fundamental a la hora de hablar de una identidad global (Giddens, 1991; Luhmann, 1991; y Beck, 1992). Existe una conciencia cada vez más extendida de los desastres ecológicos que se producen en nuestro planeta y por lo tanto se hace difícil hablar de fronteras territoriales o incluso identitarias. Sólo una comunidad global puede hacerse cargo de una cuestión que debe ser encarada globalmente.

Giddens, ante el proceso dialéctico de lo global y lo local, entiende a la persona como un sujeto que se ve forzado a elegir entre diferentes estilos de vida dentro de un gran abanico de opciones. Esto lo convierte en un sujeto reflexivo y calculador.

Por su parte, Berger y Luckmann (1997) perciben una crisis de sentido generado por el pluralismo imperante, lo que provoca una disolución de las identidades compartidas. Estos autores proponen centrar la investigación sobre la transmisión de sentido en tres ámbitos: la comunicación masiva, la comunicación cotidiana dentro de las comunidades y las instituciones intermedias que mediarían entre las grandes instituciones, las comunidades y el individuo. Estas instituciones intermedias tendrían un papel crucial pues serían los garantes de que los individuos no se sintieran como completos extraños en este mundo globalizado, y así evitar la crisis de sentido. (Berger y Luckmann, 1997: 122)

Parece sensato pensar que el riesgo global es un factor que puede ayudar a generar un sentimiento global, pero la creación de una identidad global necesita también de otros materiales simbólicos y culturales que doten de sentido a una propuesta identitaria a nivel planetario. 


\subsubsection{El papel de la economía global y los mass media}

La globalización en el ámbito económico y financiero es un hecho comprobado a lo largo y ancho del globo. Se está hoy en día tan convencido de encontrarse una Coca-Cola en Times Square, en el corazón de Manhattan, como en Pannur, un pequeño poblado del estado de Karnataka en la India, o en un puesto ambulante de los barrios de Casablanca en Marruecos. La transnacionalización de los productos y de los capitales ha creado un mapa mundial controlado en gran medida por las multinacionales que se manejan fluidamente en este mundo globalizado.

También es cierto que las grandes corporaciones mediáticas que nutren a los medios de gran parte del planeta están controladas por muy pocas manos, lo que produce un control e inducción de noticias en la dirección que estos magnates de la comunicación desean. Bien es sabido el poder que las multinacionales representan no sólo sobre los estados, sino sobre los medios de comunicación.

Algunos estudiosos como Featherstone (1992) denominan «terceras culturas» a aquellas que se verían influidas por el intercambio de bienes, informaciones, imágenes y conocimientos, vehiculados por redes de comunicación a nivel global, y dotados de cierta autonomía. En Estados Unidos se conoce estas «terceras culturas» como las «culturas populares» (popular culture).

En estos términos, se puede hablar de una homogeneización o extensión de una «cultura popular a nivel mundial». De lo que no podemos hablar es de una «identidad global» unificada y fuertemente integrada como la encontramos en las identidades nacionales. La formación de una «identidad global» a esos niveles, necesitaría de la formación de una sociedad política y una sociedad civil global, acompañada con instituciones que las legitimen. Este es uno de los sueños del cosmopolitismo.

\subsubsection{El proyecto cosmopolita}

«La humanidad tiene un destino, el de forjar una ciudadanía cosmopolita, posible en una suerte de república ética universal». (Cortina, 1999: 252)

El sueño de la identidad cosmopolita presupone para muchos un proyecto ético de ciudadanía internacional apoyado por una ampliación del «nosotros» a nivel social e identitario, y también por la necesidad de crear instituciones «cosmopolitas $»^{1}$ que posibiliten la participación democrática a escala planetaria (Beck, 1998: 152-159).

Desde un punto de vista menos pretencioso, algunos identifican a la «comunidad global» con una élite urbana y cosmopolita, que han llamado la «clase

1 Una de las propuestas planteadas a nivel mundial ha sido la de una «Autoridad Política Mundial» en Benedicto XVI, encíclica Caritas in veritate (2009). n. 21. 
media mundializada» (Levy, 1998: 364). Una élite acostumbrada a viajar, que domina el inglés, comparte modos de consumo, estilos de vida y biografías más o menos similares. En este esquema, la identidad cosmopolita se encarnaría en una persona que relativiza su pertenencia nacional y circula por todas las culturas. Este proyecto es difícilmente universalizable a todas las personas que viven en el mundo, como para pretender cimentar una «identidad cosmopolita» desde esta «élite urbana mundial».

\subsubsection{Las identidades múltiples}

Es de sobra conocida la obra de Bauman (2003) donde trata sobre la modernidad líquida. En este contexto, la identidad es entendida como destino, una identidad violentada por lo temporal, plural y abierta. Otros autores como Richard Sennett (2006 y 2008) siguiendo esta estela interpretativa exponen cómo el contexto neoliberal capitalista influye enormemente en la construcción de la configuración identitaria del sujeto.

«... ¿Cómo pueden perseguirse objetivos a largo plazo en una sociedad a corto plazo? ¿Cómo sostener relaciones sociales duraderas? ¿Cómo puede un ser humano desarrollar un relato de su identidad e historia vital en una sociedad compuesta de episodios y fragmentos? Las condiciones de la nueva economía se alimentan de una experiencia que va a la deriva en el tiempo, de un lugar a otro lugar, de un empleo a otro». (Sennett, 2006: 28)

Sennett presenta una visión pesimista de un mundo fagocitado por el capitalismo, en el que el consumo se ha convertido en la condición de posibilidad para convertirse en ciudadano. Las otras experiencias cotidianas de la vida ya no deciden, no confieren identidad.

Siguiendo también la estela interpretativa de Bauman, Amartya Sen (2007) presenta el sujeto compuesto por un conjunto de identidades que peregrinan por caminos de flexibilidad, que se acomoda según los contextos, y que carece de vínculos estables en el tiempo.

Estas identidades múltiples en ocasiones entran en conflicto en el interior del mismo sujeto. En ocasiones los intereses contrapuestos del ámbito local y nacional hace que la propia identidad étnica presente conflictos con la identidad nacional (Smith, 1992b: 59).

En otro orden de cosas, me parece muy sugerente la distinción que Maffesoli (2002) realiza entre individuo y persona cuando desarrolla su concepto de tribalismo (lo que a mi modo de ver más tarde describirá Castells como identidad de resistencia).

«Se trata pues, con las correspondientes consecuencias sociológicas que ello conlleva, de un desplazamiento que va del individuo con una identidad estable, que ejerce su función dentro de conjuntos contractuales, hacia la persona, con identificaciones múltiples, que desempeña papeles en tribus determinadas por sus elementos afectivos». (Maffesoli, 2002: 238) 
Para este autor, se debe abandonar el término individuo porque no es capaz de adecuarse a la dinamicidad y flexibilidad que vive el ser humano actual. El término individuo haría referencia a una identidad única y homogénea. Sin embargo, la persona, siguiendo su sentido etimológico «personae» es una máscara que tiene diversos papeles. De este modo, la persona utilizará distintas máscaras dependiendo el contexto, la «tribu» en la que se encuentre en cada momento. Esta multiplicidad de máscaras y su función según los momentos, ayudará a no vivir desde la esquizofrenia en cuanto a la lógica de la identidad, pues lo que estará en juego es la función, el papel que se juega en cada uno de los distintos grupos en los que se participa.

\subsubsection{Un estudio empírico sobre las identidades}

Para concluir este apartado, me parece interesante contrastar estos tres tipos de identidades con la percepción que de ellas tenemos las personas. Pipa Norris, una socióloga de la Universidad de Harvard, realizó en el año 2000 un estudio sobre la relación entre identidad y pertenencia territorial en la década de los 90 del siglo pasado². Norris se apoyó en la principal fuente de estadísticas comparadas sobre actitudes, valores y opiniones, el World Values Survey, el centro de mayor prestigio e influencia en el ámbito de las encuestas de opinión y dependiente de la Universidad de Michigan.

Las tres identidades sobre las que se realizó dicha encuesta fueron la identidad mundial/continente (cosmopolita), la identidad nacional y la identidad locall regional. Parece curioso como en plena era de la globalización sólo el 15\% de las personas se identifica con el ámbito global, el mundo o su continente, frente al $47 \%$ que se identifica en primer lugar con su región o localidad. Por su parte, un 38\% se refieren en primer lugar al estado-nación. Si comparamos los datos por edades, el estudio nos dice que los jóvenes son más cosmopolitas que los viejos. En cuanto al sexo, las mujeres tienden a identificarse un poco más que los hombres con su localidad o región. Por su parte, los hombres se identifican ligeramente más que las mujeres con la nación y el mundo.

Un dato que parece marcar la diferencia es la educación. Las personas con una formación más baja se identifican con el ámbito local en un 64\%, por un $40 \%$ de las personas con niveles de formación más altos. Aunque el desfase en la percepción de la identidad nacional también es muy amplio, es en la conciencia mundial donde las personas con alta educación duplican ampliamente a las personas con una formación más baja. Del mismo modo, es también significativa la lectura de los datos en las poblaciones por número de habitantes. La conciencia hacia la identidad global casi se duplica en las grandes ciudades, frente a los núcleos de población de menos de 2000 habitantes.

2 La tabla que se presenta en este apartado y el estudio de la profesora Norris se puede consultar en el artículo de Pippa NorRIs (2003). 
Tabla 1

Relación entre identidad y pertenencia territorial (Norris)

\begin{tabular}{|c|c|c|c|c|}
\hline & & $\begin{array}{c}\text { World- } \\
\text { Continent }\end{array}$ & National & $\begin{array}{c}\text { Local- } \\
\text { Regional }\end{array}$ \\
\hline ALL & & 15 & 38 & 47 \\
\hline \multirow[t]{3}{*}{ Type of Society } & Post-Industrial & 15 & 41 & 44 \\
\hline & Post-Communist & 16 & 32 & 53 \\
\hline & Developing & 14 & 37 & 49 \\
\hline \multirow[t]{7}{*}{ Cohort } & 1905-1914 & 6 & 33 & 62 \\
\hline & 1915-1924 & 10 & 35 & 55 \\
\hline & 1925-1934 & 10 & 38 & 53 \\
\hline & 1935-1944 & 11 & 38 & 51 \\
\hline & 1945-1954 & 19 & 37 & 44 \\
\hline & $1955-1964$ & 17 & 35 & 48 \\
\hline & $1965-1978$ & 21 & 34 & 44 \\
\hline \multirow[t]{10}{*}{ Continent } & North Americ a & 16 & 43 & 41 \\
\hline & South America & 17 & 37 & 45 \\
\hline & North Europe & 11 & 36 & 53 \\
\hline & North-Western Europe & 13 & 25 & 62 \\
\hline & South-Western Europe & 13 & 23 & 64 \\
\hline & Eastern Europe & 8 & 34 & 58 \\
\hline & Former Soviet Union & 15 & 32 & 53 \\
\hline & Middle East & 12 & 49 & 39 \\
\hline & Asia & 13 & 32 & 55 \\
\hline & A.frica & 9 & 41 & 49 \\
\hline \multirow[t]{2}{*}{ Education } & Highest & 18 & 42 & 40 \\
\hline & Lowest & 7 & 29 & 64 \\
\hline \multirow[t]{2}{*}{ Gencler } & Men & 16 & 40 & 45 \\
\hline & Women & 14 & 36 & 49 \\
\hline \multirow[t]{2}{*}{ Size of town } & Low (Less than 2000) & 11 & 34 & 55 \\
\hline & High (More than $500 \mathrm{~K}$ ) & 21 & 36 & 43 \\
\hline \multirow[t]{9}{*}{ Type of Culture } & Northern European & 12 & 36 & 53 \\
\hline & English & 19 & 41 & 41 \\
\hline & Catholic European & 13 & 24 & 64 \\
\hline & Confucian & 5 & 44 & 52 \\
\hline & Central European & 7 & 33 & 60 \\
\hline & Soviet & 16 & 31 & 53 \\
\hline & Latín American & 8 & 50 & 43 \\
\hline & South-East Asian & 8 & 29 & 63 \\
\hline & A.frican & 9 & 41 & 49 \\
\hline \multirow[t]{3}{*}{ Post-Materialism } & Materialist & 12 & 38 & 50 \\
\hline & Mixed & 16 & 39 & 45 \\
\hline & Post-Materialist & 20 & 37 & 43 \\
\hline \multirow[t]{3}{*}{ Type of Democracy } & Free & 16 & 39 & 45 \\
\hline & Partly Free & 15 & 32 & 53 \\
\hline & Non-Free & 10 & 32 & 58 \\
\hline
\end{tabular}

Note: "To which, of these geographical groups would you say you belong first of all?

- The locality or town where you live

- The state or region of the country in which you live

- Your country [The U.S, France, etc.] as a whole*

- The continent in which you live [North America/Europe/Asia/Latin America, etc]*

- The world as a whole"

Source: World Values Surveys combined waves 1990-91 and 1995-7, weighted date (N.147319) 


\section{IDENTIDADES COLECTIVAS: LEGITIMADORA, DE RESISTENCIA Y PROYECTO}

La idea de «sujeto racional y calculador», y esa vuelta a la reflexividad del sujeto tan extendida, perdiendo campo en su ámbito colectivo y social en cuanto a la creación de identidad, han sido criticadas por autores como Castells (2000). Este autor se basa en la idea de «sujeto autor» que propone Touraine (1995: 29): «Denomino sujeto (...) al deseo de ser un individuo, de crear una historia personal, de dar sentido a todo el conjunto de experiencias de la vida individual». Un sujeto que mediante las identidades colectivas desafía a la individualización creciente fruto de la globalización y a los cambios producidos en la posmodernidad.

Castells presenta la sociedad red, como una nueva sociedad creada a partir de las nuevas tecnologías de la información y la reestructuración del capitalismo. Esta sociedad red se caracterizaría por la globalización en el ámbito económico, la organización en redes, la inestabilidad y flexibilidad del mercado de trabajo y una cultura virtual cimentada a través de los medios de comunicación masivos (Castells, 2000: 23).

Es en este contexto donde Castells propone la identidad colectiva, como un desafío y una respuesta a la globalización y el cosmopolitismo. Su propuesta de construcción identitaria está basada principalmente en dos conceptos clave: el «sujeto actor» y la "construcción de sentido». Sobre el primero ya hemos apuntado desde la perspectiva de Touraine.

Sobre el segundo, Castells definiría la identidad como un "proceso de construcción de sentido atendiendo a un atributo cultural, o un conjunto relacionado de atributos culturales, al que se da prioridad sobre el resto de las fuentes de sentido» (Castells, 2000: 28-29). Este autor hace hincapié en el carácter interaccional y social de la identidad. La identidad no se construye a partir de uno mismo, sino a partir del grupo, es decir, de los valores, normas, representaciones y creencias compartidas. Son estos elementos grupales los que guían la acción de los individuos.

El sentido sería «como la identificación simbólica que realiza un actor social del objetivo de su acción». Esta idea de actor con sentido social (Weber, 1934) abriría para Castells la posibilidad de construcción de identidades colectivas a partir de la acción colectiva.

En el camino para poner luz en la construcción de la identidad, Castells propone una diferencia entre roles e identidades. Mientras los roles se definen por normas establecidas por organizaciones e instituciones, las identidades, por su parte, son construcciones de sentido realizadas por los individuos a través de un proceso de individuación y autodefinición. Estos roles pueden convertirse en normas cuando son interiorizados por los actores, construyendo su sentido en torno a ellos.

De este modo, los roles se encargarían de organizar las funciones, mientras que la identidad se ocuparía de organizar el sentido. Los roles son otorgados, mientras que las identidades necesitan ser construidas. 
Castells, como se ha apuntado anteriormente, centra su análisis en la identidad colectiva por encima de la identidad individual: «quién construye la identidad colectiva, y para qué, determina en buena medida su contenido simbólico y su sentido para quienes se identifican con ella o se colocan fuera de ella.» (Castell, 2000: 29)

Analizando la actual sociedad de la información, Castells diferencia tres tipos de identidades: legitimadora, de resistencia y proyecto. La identidad legitimadora es planteada por las instituciones dominantes de la sociedad para extender su dominación frente a los actores sociales. La identidad de resistencia es generada por aquellos actores sociales que se encuentran en posiciones marginales y devaluadas desde la posición dominante, por lo que constituyen trincheras de supervivencia y resistencia. Por último, la identidad proyecto se da cuando los actores sociales construyen una nueva identidad a partir de los materiales culturales disponibles, redefiniendo su posición en la sociedad y buscando la transformación de la estructura social.

Estos tres tipos de identidad tienen que entenderse dentro de su contexto histórico, es decir, dentro de unas coordenadas espaciotemporales. No podemos entender a priori ninguna identidad como progresiva o regresiva, sino es dentro de su contexto. Ni tampoco podemos hacer extrapolaciones a momentos o lugares distintos.

\section{LA IDENTIDAD Y EL MODELO DE INTEGRACIÓN MIXTO}

La construcción de la propia identidad es un proceso complejo, multidimensional, abierto y flexible que acompaña el proceso vital de todo ser humano. En esta era de la globalización donde los intercambios comerciales, de información y la movilidad humana son elementos que se imponen en nuestra vida cotidiana, se hace necesario hoy más que nunca preguntarse por cómo nuestras sociedades viven los procesos de integración, la gestión de la diversidad y la «recreación» de identidades.

Se han encontrado en el modelo de construcción de identidades de Castells (2000), junto al estudio realizado por Norris (2003) y los modelos de integración clásicos de personas inmigrantes una sintonía y unos espacios comunes que se piensa pueden ayudar a desarrollar el modelo de integración mixto. Seguidamente se expondrá esos atributos comunes para más tarde analizar el modelo mixto de integración, basado en las conductas mayoritarias y minoritarias (Segundo, 1973). 
Tabla 2

Identidad y modelo de integración mixto (MIM).

\begin{tabular}{|c|c|c|c|c|}
\hline $\begin{array}{l}\text { NORRIS } \\
\text { (World Values }\end{array}$ & $\begin{array}{l}\text { CASTELLS } \\
\text { (El poder de la }\end{array}$ & $\begin{array}{l}\text { Modelos de } \\
\text { integración }\end{array}$ & \multicolumn{2}{|c|}{ Modelo de integración mixto } \\
\hline $\begin{array}{l}\text { Identidad } \\
\text { Nacional } \\
\text { (Estados- } \\
\text { nación) }\end{array}$ & $\begin{array}{l}\text { Identidad } \\
\text { Legitimadora }\end{array}$ & $\begin{array}{c}\text { Modelo } \\
\text { Asimilacionista }\end{array}$ & $\begin{array}{c}\text { Conductas } \\
\text { «Asimilacionistas» }\end{array}$ & \multirow[t]{2}{*}{$\begin{array}{l}\text { Conductas } \\
\text { Mayoritarias }\end{array}$} \\
\hline $\begin{array}{l}\text { Identidad Local } \\
\text { (Étnica) }\end{array}$ & $\begin{array}{l}\text { Identidad de } \\
\text { Resistencia }\end{array}$ & $\begin{array}{c}\text { Modelo } \\
\text { Multiculturalista }\end{array}$ & $\begin{array}{c}\text { Conductas } \\
\text { «Multiculturales» }\end{array}$ & \\
\hline $\begin{array}{c}\text { Identidad } \\
\text { Global } \\
\text { (Cosmopolita) }\end{array}$ & $\begin{array}{l}\text { Identidad } \\
\text { Proyecto }\end{array}$ & $\begin{array}{c}\text { Modelo } \\
\text { Integracionista }\end{array}$ & $\begin{array}{l}\text { Conductas } \\
\text { «Proyecto» }\end{array}$ & $\begin{array}{l}\text { Conductas } \\
\text { Minoritarias }\end{array}$ \\
\hline
\end{tabular}

Fuente: elaboración propia.

\subsection{Identidad y modelos de integración}

Existen modelos unidimensionales que, simplificando en buena medida la realidad, han logrado plantear propuestas para construir la identidad y gestionar la diversidad en nuestras sociedades. A mi modo de ver, se pueden encontrar puntos comunes en la propuesta de identidad nacional (estado-nación), de identidad legitimadora y en el modelo asimilacionista de integración.

Algunos de los elementos compartidos en estos tres planteamientos son:

1.- Tendencia a la homogeneización de toda la sociedad de manos de la cultura dominante.

2.- Imposición de unos patrones, valores e identidad común que tienden a institucionalizarse.

3.- Creación de una historia y modelo educativo común que tiende a imponerse en el territorio del estado.

4.- Simplificación de la realidad y de la vida social.

5.- Dificultades para gestionar la diversidad cultural, lingüística, etc.

6.- Fuerte presión del estado-nación y de las instituciones que lo legitiman sobre los individuos, especialmente en la gestión de la diversidad cultural con las comunidades migrantes.

7.- Mayor igualdad en cuanto al acceso a derechos básicos vía ciudadanía.

En el otro extremo, se han desarrollado en nuestras sociedades modelos que han atendido fuertemente a la diversidad sobre todo en lo que se refiere a lo étnico-cultural. De este modo, se puede hablar de las construcciones de 
identidades locales y de resistencia, así como de modelos de gestión de la diversidad «multiculturales». Muchos de estos modelos han surgido como reacción al monolitismo unidimensional dentro del ámbito cultural.

Con respecto a sus elementos comunes, se pueden encontrar:

1.- Tendencia a la heterogeneidad cultural entre las culturas que conviven en un mismo espacio territorial.

2.- Respeto de la diversidad de patrones y valores de las personas que comparten un mismo espacio geográfico.

3.- Convivencia de tradiciones e historias diversas.

4.- Acogida de lo diverso dentro de la realidad y vida social.

5.- Facilidad para gestionar la diversidad cultural, lingüística, etc.

6.- Fuerte presión de lo étnico y del grupo de iguales en cuanto a la imposición cultural de los propios patrones en el interior del grupo.

7.- Dificultad para la gestión del acceso a derechos básicos comunes y creación de desigualdades y de asimetrías de poder dentro de las diversas etnias que conviven en el mismo territorio. Este elemento tiene una importancia notable sobre las comunidades migrantes y la vulnerabilidad de derechos y precariedad en diversos ámbitos de las perspectivas socioeconómicas.

Asimismo, se están planteando modelos de construcción de la identidad intentando acoger lo diverso, con un igual acceso a los derechos básicos de todos los seres humanos y el respeto a lo multicultural. Son modelos que intentan dar un paso más en la construcción y transformación de nuestras sociedades. Modelos que buscan acoger la realidad de manera multidimensional, ampliando el espectro de lo «cultural» — que define los modelos anteriores-, para acoger otros aspectos tan importantes como son la faceta socioeconómica y política de nuestras sociedades. En este camino se abrirían paso los modelos de identidad cosmopolita, de identidad proyecto y el modelo «integracionista» ${ }^{3}$.

¿Cuáles son algunos elementos comunes?

1.- Construcción de una nueva identidad a partir de los materiales culturales disponibles. Una nueva identidad que va más allá de la imposición o el respeto a la diversidad cultural.

2.- Construcción de procesos de integración multilaterales. Los procesos de integración no sólo conciernen a la comunidad que emigra, sino a la comunidad de la sociedad de acogida o recepción. Si además se añaden la fuerte diversidad de comunidades que emigran en un mismo territorio, ya no se podría hablar de bilateralidad, sino de multilateralidad.

3 Hoy en día muchos expertos denominan a estas «nuevas propuestas» como «interculturales». A mi modo de ver la misma terminología impide salir del monolitismo cultural y no ayuda a la inclusión de las otras facetas de la integración («lo sociopolítico y legal»). Por esa razón, más adelante denominaré a estas conductas del modelo de integración mixto (MIM), como «conductas proyecto». 
3.- Acogida de la integración como un proceso complejo y multidimensional. La integración pasa por acoger los elementos tanto culturales, como sociopolíticos y legales. Sin el pleno acceso a los derechos en condiciones de igualdad, de poco sirve el respeto y la aceptación de lo cultural (si bien es un gran paso).

4.- Búsqueda de la transformación social. La recreación y construcción del posicionamiento en la sociedad, buscando la transformación de la estructura social.

\subsection{El modelo de integración mixto}

Mi propuesta sería un modelo de integración mixto según el planteamiento de conductas mayoritarias y minoritarias de Juan Luis Segundo (1973). Para este autor, el ser humano tiene una energía limitada que utiliza para primar aquellas actividades que le generan un mayor valor añadido en su vida. Estas conductas son las que Segundo describe como conductas minoritarias. Por otro lado, las conductas mayoritarias, serían aquellas que se realizan de forma más mecánica y en las que no se emplea tanta energía vital.

4.2.1. Conductas mayoritarias: «conductas asimilacionistas» $\mathrm{y}$ «conductas multiculturales»

- «Conductas asimilacionistas»

Según las conductas mayoritarias y según una «economía» de energía, habrá conductas en la vida que se tenderán a mecanizar. Éstas no ocupan un papel central en la realidad de la vida actual, o se imponen de tal manera al sujeto que no se va a emplear mucha energía para ser discernidas o dedicarles un tiempo extra. Lo mismo ocurre cuando una persona emigra. Existirán unas conductas mayoritarias en las cuales unas personas no van a perder muchas energías. Así habrá facetas o dimensiones en el proceso de integración en los cuales se esté más dispuesto a «asimilarse». No se va a emplear mucho tiempo en reflexionar y decidir. Puede haber dimensiones como la búsqueda de empleo, el idioma, el consumo... donde según los contextos una persona puede tender a asimilarse. « ¿Voy a pensar si es bueno o no obtener un empleo, o aprender un idioma? Muchas veces esta misma realidad se me impone».

\section{- «Conductas multiculturales»}

En el otro extremo de estas conductas mayoritarias estarían las dimensiones en las que tampoco se va a perder mucho tiempo en discernir pues se imponen como constitutivas de la propia identidad o simplemente no se está dispuesto a negociar con ellas. Son dimensiones que yo respeto en los otros, y también en las que espero un respeto mutuo (multiculturalismo). En algunas personas estas conductas pudieran ser las celebraciones populares, la práctica religiosa, cierto tipo de consumo o vestimenta étnica, etc. 
Generalmente, estas dimensiones ayudan a sentirse unido a las propias raíces, a la identidad local o étnica. Además existe en muchos casos una fuerte presión familiar, tribal o grupal que imprime un fuerte influjo sobre el sujeto.

En la experiencia de una persona que emigra, en ocasiones estas conductas multiculturales se fortalecen, debido a la necesidad de seguir conectada a sus raíces y a los que dejó atrás. En otras ocasiones algunos de estos elementos étnicos se debilitan debido a diferentes causas, por ejemplo la falta de conexiones con el grupo étnico de origen en la sociedad de acogida. De todos modos, algunas dimensiones que para unas personas pueden ser «conductas multiculturales», para otras se pueden convertir en "asimilacionistas» o pasar a ser "conductas proyecto».

\subsubsection{Conductas minoritarias: «conductas proyecto»}

Junto a estas dimensiones de síntesis fáciles, estarían otras que se constituyen como conductas minoritarias. Nuestra energía limitada se empleará en aquellas facetas que ayudarán a construir algo nuevo, algo por lo que merezca la pena vivir. Es decir, emplearía las propias energías en redefinir la posición que se ocupa en la sociedad, buscando un marco común para construir una nueva convivencia, una nueva ciudadanía, un proyecto común, como paso para una verdadera transformación de la estructura social. Estas conductas tendrían mucho que ver con la idea de proceso, de multidimensionalidad y de adaptación mutua, entre otros.

¿Qué características sería deseable que tuviera una persona en aras a construir el proceso y la transformación social?

1.- Sería una persona con «subjecto», como diría Ignacio de Loyola en la anotación 18 de sus Ejercicios Espirituales (Loyola, 1991).

2.- Capaz de vivir con cierta libertad frente a las presiones del grupo (étnicas) y de la nación (estado-nación), así como de otras presiones «cosmopolitas» centradas en el consumo y los mass media.

3..- Capaz de asumir un riesgo razonable y de apostar por un proyecto común de futuro.

4.- Con flexibilidad para vivir la diversidad y aceptar las limitaciones y bondades de cada manifestación cultural.

5.- Con cierta capacidad para gestionar la propia ambigüedad.

6.- Con habilidades para trabajar en equipo, respetando la diversidad.

7.- Con madurez y capacidad de reflexionar críticamente y de decidir, fruto de las síntesis difíciles de las que hablaba Segundo.

\section{CAMINANDO HACIA LA INTEGRACIÓN Y LA COHESIÓN SOCIAL}

La construcción de la identidad es un elemento constitutivo en el desarrollo vital de la persona y de los colectivos. En nuestro mundo actual globalizado y 
diverso, este elemento cobra una relevancia nuclear. Es en el día a día donde se juegan muchas de las dinámicas identitarias, en las que entrelazan procesos de identidad personal y colectiva, tendencias más estáticas y dinámicas. Lo diverso a veces genera miedo, incertidumbre, nos hace salir de nuestra zona de confort hacia un espacio nuevo de diálogo, de riesgo. En ese contexto, corremos el riesgo de cerrar filas y de apoyarnos en nuestras seguridades, levantar muros que aíslen y que generen mecanismos de exclusión.

Pero lo diverso también nos abre a lo más esencial del ser humano. Cada persona va construyendo su identidad en relación con los demás, como personas únicas. La diversidad se convierte en condición de posibilidad para la recreación de una identidad que es múltiple, abierta, flexible y compleja.

¿Qué ocurre entonces en nuestro mundo occidental donde «lo diverso» se ha convertido en arma arrojadiza en el ámbito político y de exclusión social? ¿Por qué el populismo y el cerrar filas está tomando una relevancia en algunas sociedades como la estadounidense (Trump), en Reino Unido (Brexit), el aumento de la ultraderecha nacionalista en varios países europeos, entre ellos Francia (Le Pen)?

Es lícito tener miedo ante lo nuevo, lo diverso. De hecho, muchas de estas tendencias escuchan este temor que busca simplificar nuestra vida, nuestra cultura, hacia un horizonte homogéneo y unidimensional. Miran el corto plazo y una solución «fácil». Si el construir muros, además de simplificar nuestra vida, ayudara a crecer como sociedad este elemento sería la solución final. Pero la diversidad es algo constitutivo de nuestra existencia, lo queramos o no.

La era de la globalización tiene que integrar estas tres maneras de construcción de identidad (local, nacional y global) que de una u otra manera conviven en nuestra sociedad y en cada uno de nosotros. Intentar reducir el espectro, crea tensiones y excluye, como estamos observando actualmente. Una sociedad que vive desde lo global, desde las grandes ciudades, que se apoya en una «élite urbana y cosmopolita», está obviando y dejando atrás a miles de millones de personas en el camino. Esta tendencia unida a una globalización económica y financiera que realmente «no conoce fronteras», desregulada y que excluye y atenta contra los derechos laborales de las personas, entre otros, empuja a posiciones como las que vivimos en diversos rincones de nuestro mundo. El análisis de Norris (2003) aporta mucha luz en este punto. ¿Quiénes están apoyando en gran medida estos movimientos populistas? Muchas de las personas que no viven en las grandes urbes, que no han accedido a una educación superior, que por edad se han quedado algo atrás en la brecha digital y en un mundo interconectado, de clase media baja y a las cuales las crisis económicas han desplazado del mercado laboral.

Asimismo, unos colectivos que les ha tocado vivir la diversidad, sin estar del todo preparados para ello tanto por formación, como por conectividad, entre otras razones. Son muchas de estas comunidades las que soportan en sus barrios un alto grado de diversidad cultural y étnica, desde una precariedad sobrevenida en el ámbito social y económica. Además, desde contextos donde el recorte de derechos sociales en las últimas décadas se ha hecho más patente, 
y donde el estado del bienestar se ha ido resquebrajando. Viviendo un contexto mundial donde hay muchos intereses que intentan asociar migración con terrorismo y conflicto social (Barciela, 2019; Caldwell, 2017). En ese contexto, es más fácil buscar chivos expiatorios en el otro, el diferente 4 .

Surgen así colectivos que ya no aguantan más a un "establishment», a una casta política, económica y social que solo mira por sus intereses y por perpetuarse en el poder y en sus cargos, sea cual sea el color político. Todo esto unido en muchos contextos de continuos casos de corrupción, que salpican no sólo a políticos y empresarios, sino a algunas monarquías como a la española.

¿La bandera de la «identidad nacional» es la solución a todos nuestros males? A corto plazo puede ser un pequeño respiro desde un punto de vista económico o financiero, pero a mi modo de ver, será pan para hoy y hambre para mañana. Construir muros, cerrar fronteras creo que no es la solución, pero plantea cuestiones que no podemos obviar. Nuestro mundo globalizado necesita reglas de juego a distintos niveles, para que puedan convivir los «tres tipos de identidades», y para que se tomen en cuenta las necesidades y la voz del conjunto social. De otro modo, seguiremos perpetuando la exclusión, los conflictos, la pobreza y una huida hacia adelante de una «élite cosmopolita mundial» que vive en una burbuja «irreal e inalcanzable» para miles de millones de personas. Como diría Castells (2000), seguiremos perpetuando una identidad legitimadora.

Dando un paso más, ¿cómo afecta la creación de identidad a la integración y a la cohesión social? Como venimos compartiendo, la gestión de la diversidad en clave de identidad es un fenómeno completo y abierto, en el que conviven procesos tanto étnicos, nacionales, como globales. Estos tres niveles de identidades tienen a mi modo de ver un paralelismo como los modelos teóricos de integración. En este contexto, es desde donde se puede concluir que no hay un solo modelo de integración que logre explicar de forma completa el proceso de integración o incorporación de una persona migrante. Como tampoco sería explicable la creación de identidad de una persona si sólo viéramos de forma unidimensional la identidad local, nacional o cosmopolita. En la práctica se da un modelo de integración mixto con actitudes y conductas tanto asimilacionismas, multiculturales, y de proyecto.

Apoyados en Segundo, se podría decir que la persona migrante se incorpora al proceso de integración desde unas conductas mayoritarias y minoritarias que la constituyen. Estas conductas no serían universalizables para todos los individuos. Tal vez una persona viva los hábitos de consumo como un elemento en el que es fácilmente asimilable (comienza pronto a comer la tortilla de patatas y la paella, y a cocinarla en casa dejando los platos típicos de su

4 En este sentido, Huntington en su libro ¿Quiénes somos? Desafíos de la identidad nacional estadounidense (2004), plantea que la probabilidad de que los estadounidenses se sientan identificados con su nación aumenta cuando consideran que ésta se encuentra amenazada, pero en el momento en que esta sensación de amenaza o peligro pierde intensidad desaparece entre los ciudadanos la prioridad de la identidad nacional. 
cultura de referencia), pero para otros puede ser un elemento que no tiene discusión. Ni se plantearán abandonar sus pupusas, su cuscús o su ceviche, sin incorporar apenas variaciones en su dieta, buscando todos los ingredientes cueste lo que cueste. Sin embargo, habrá otros que se planteen disfrutar de sus platos preferidos en sus países de origen, pero sin miedo a introducir nuevos ingredientes, incorporando a su dieta nuevos platos, nuevos sabores y olores, haciéndola dialogar con su cocina y elaborando una nueva síntesis.

Llevado al extremo se puede decir que hay tantos procesos de integración como personas que emigran. Ningún proceso de integración personal es igual a otro. Dicho esto, se pueden dar ciertas similitudes entre individuos en los procesos de incorporación.

La idea central es recalcar un modelo de integración mixto que reconoce elementos de los modelos clásicos de integración, pero sin encasillarse exclusivamente en ninguno de ellos. Ninguno por si solo es capaz de explicar, describir y dar sentido a un proceso multidimensional, abierto, como es el proyecto de incorporación a una nueva sociedad, y las dinámicas que este proceso genera. (Sen, 2011: 24).

Estamos llamados a convivir, a enriquecernos de lo diverso. Lo diverso nos abre a lo más esencial del ser humano. Cada persona va construyendo su identidad en relación con los demás, como personas únicas. La diversidad se convierte en condición de posibilidad para la recreación de una sociedad que es múltiple, abierta, flexible y compleja. Lo diverso en cierta manera es un horizonte, una cierta utopía, pero que se va haciendo cuerpo, camino, proceso y realidad.

\section{BIBLIOGRAFÍA}

Ares, A. (2017). La rueca migratoria. Tejiendo historias y experiencias de integración. Madrid: Universidad Pontificia Comillas.

Barciela, S. (2019). Arde la ciudad: conflicto en barrios. Madrid: Universidad Pontificia Comillas.

Bauman, Z. (1991). "Modernidad y ambivalencia» en Beriain J. (comp.) (1996) Las consecuencias perversas de la modernidad. Barcelona: Anthropos.

Bauman, Z. (2003). Modernidad líquida. México: Fondo de Cultura Económica.

Baumann, G. (2001). El enigma multicultural. Un replanteamiento de las identidades nacionales, étnicas y religiosas. Barcelona: Paidós.

Beck, U. (1992). Risk society: Towards a new modernity. London: Editorial Sage.

Beck, U. (1998). ¿Qué es la globalización? Falacias del globalismo, respuestas a la globalización. Barcelona: Paidós.

Beck, U. (2002). La sociedad del riesgo global. Madrid: Siglo XXI.

Bellah, R. (1985). Habits of the heart: individualism and commitment in American life: with a new preface. University of California Press.

Benedicto XVI (2009). Caritas in veritate. Ciudad del Vaticano. El texto puede consultarse en www.vatican.va.

Berger, P. y Luckmann, T. (1986). La construcción social de la realidad. Buenos Aires: Amorrortu. 
Berger, P. L. y Luckmann, T. (1997). Modernidad, pluralismo y crisis de sentido. La orientación del hombre moderno. Barcelona: Paidós.

Buber, M. (1969). Yo y Tú. Buenos Aires: Nueva Visión.

Cadwell, C. (2017). «La inmigración levanta pasiones de identidad nacional». Expansión, actualidad económica. 01/02/2017.

Castells, M. (2000). La era de la información. Economía, sociedad y cultura. Vol. 2: El poder de la identidad. Madrid: Alianza.

Cortina, A. (1999). Ciudadanos del mundo. Hacia una teoría de la ciudadanía. Madrid: Alianza.

Featherstone, M. (ed.) (1992). Global Culture. London: Sage Publications.

Fitzgerald, T. K. (1993). Metaphors of Identity. A Culture-Communication Dialogue. Albany: State University of New York Press.

Gellner, E. (1988). Naciones y nacionalismo. Madrid: Alianza.

Giddens, A. (1991). Modernidad e identidad del yo. El yo y la sociedad en la época contemporánea. Barcelona: Península.

Huntington, S. P. (2004). ¿Quiénes somos?: Los desafíos a la identidad nacional estadounidense. Barcelona: Paidós.

Labrador, J. (2001). Identidad e inmigración: un estudio cualitativo con inmigrantes peruanos en Madrid. Madrid: Universidad Pontifica Comillas.

Lévy, J. (1998), Vers une société civile mondiale?, en: Jean Claude Ruano-Borbalan, L'identité, París: Éditions Sciences Humaines: 363-368.

Loyola, I. (1991). Ejercicios espirituales. Bilbao: Mensajero.

Luhmann, K. (1991). Sociología del riesgo. Guadalajara (México): Universidad Iberoamericana - Universidad Guadalajara. Col. Laberinto de cristal.

Maffesoli, M. (2000). «Posmodernidad e identidades múltiples» en Sociológica. No. 43, mayo-agosto.

Maffesoli, M. (2001). El ocaso del individualismo en las sociedades posmodernas. México D.F.: S.XXI.

Maffesoli, M. (2002). «Tribalismo posmoderno. De la identidad a las identificaciones», en Chihu, Aquiles (coord). Sociología de la identidad. UAM Iztapalapa/Porrúa, México, 2002.

Martínez, J. (2007). Ciudadanía, migraciones y religión: un diálogo ético desde la fe cristiana. Madrid: Universidad Pontificia Comillas.

Noam, C. (2002). Los límites de la globalización. Barcelona: Ariel Practicum.

Norris, P. (2003). «Global Governance and Cosmopolitan Cities.» Global Transformations Reader: An Introduction to the Globalization Debate (2nd edition). Ed. David Held and Anthony McGrew. Polity: 287-297.

Robertson, R. (2003). «Glocalización: tiempo-espacio y homogeneidad-heterogeneidad». Cansancio del Leviatán: problemas políticos de la mundialización. Madrid: Trotta.

Segundo, J. L. (1973). Masas y minorías. En la dialéctica divina de la liberación. Buenos Aires: La aurora.

Sen, A. (2007). Identidad y Violencia: La Ilusión del Destino. Buenos Aires y Madrid: Katz.

Sen, A. (ed.) (2011). Peace and Democratic Society. Cambridge, UK: Open Book Publishers.

Sen, A. (2000). Development as freedom. New York: Anchor Books.

Sennett, R. (2006). La corrosión del carácter. Barcelona: Anagrama, novena edición.

Sennett, R. (2008). La cultura del nuevo capitalismo. Barcelona: Anagrama, tercera edición 
Smith, A. D. (1992a). «Towards a Global Culture?», in: Mike Featherstone, Global Culture. London: Sage Publications: 171-191.

Smith, A. D. (1992b). «National Identity and the Idea of European Unity». International Affairs. Vol. 68.

Smith, A. D. (1997). La identidad nacional, Madrid: Trama editorial.

Touraine, A. (1995). «La formation du sujet». En F. Dubet \& M. Wieviorka (dir.). Penser le sujet. Autour de l'ouvre d'Alain Touraine. Colloque de Cerisy. Paris: Fayard.

Weber, M. (2003 [1934]). La ética protestante y el espíritu del capitalismo. México: FCE.

Universidad Pontificia Comillas

Alberto Ares Mateos

alares@comillas.edu

[Artículo aprobado para publicación en enero de 2018] 